“(C) 2018 IEEE. Personal use of this material is permitted. Permission from IEEE must be obtained for all other uses, in any current or future media, including reprinting/republishing this material for advertising or promotional purposes, creating new collective works, for resale or redistribution to servers or lists, or reuse of any copyrighted component of this work in other works." 


\title{
Learning from Mistakes: \\ An Empirical Study of Elicitation Interviews performed by Novices
}

\author{
Muneera Bano ${ }^{1,2}$, Didar Zowghi ${ }^{2}$, Alessio Ferrari ${ }^{3}$, Paola Spoletini ${ }^{4}$, Beatrice Donati ${ }^{5}$ \\ ${ }^{1}$ Swinburne University of Technology, Melbourne, Australia \\ ${ }^{2}$ University of Technology Sydney, Australia \\ ${ }^{3}$ CNR-ISTI, Pisa, Italy \\ ${ }^{4}$ Kennesaw State University, GA, USA \\ ${ }^{5}$ University of Florence, DILEF, Florence, Italy \\ mbano@swin.edu.au, Muneera.Bano@uts.edu.au,Didar.Zowghi@uts.edu.au, \\ alessio.ferrari@isti.cnr.it, pspoleti@kennesaw.edu, beatrice.donati@unifi.it
}

\begin{abstract}
Context] Interviews are the most widely used elicitation technique in requirements engineering. However, conducting effective requirements elicitation interviews is challenging, due to the combination of technical and soft skills that requirements analysts often acquire after a long period of professional practice. Empirical evidence about training the novices on conducting effective requirements elicitation interviews is scarce. [Objectives] We present a list of most common mistakes that novices make in requirements elicitation interviews. The objective is to assist the educators in teaching interviewing skills to student analysts. [Research Method] We conducted an empirical study involving roleplaying and authentic assessment with 110 students, teamed up in 28 groups, to conduct interviews with a customer. One researcher made observation notes during the interview while two researchers reviewed the recordings. We qualitatively analyzed the data to identify the themes and classify the mistakes. [Results and conclusion] We identified 34 unique mistakes classified into 7 high level themes. We also give examples of the mistakes made by the novices in each theme, to assist the educationists and trainers. Our research design is a novel combination of well-known pedagogical approaches described in sufficient details to make it repeatable for future requirements engineering education and training research.

Index Terms - Requirements Elicitation Techniques, Interviews, Requirements Engineering Education
\end{abstract}

\section{INTRODUCTION}

Interview techniques have been used in a variety of fields, from journalism to anthropology, to learn about the conscious or tacit ideas, concepts and knowledge that people carry inside their minds on any phenomenon [1]. An interview is a communicative event in which an interviewer asks questions to reach to the reality of a phenomenon conceived inside the mind of the interviewee.

Requirements elicitation aims at learning and discovering the needs of the stakeholders of the system [2]. The information gathered during requirements elicitation needs to be correct, complete and unambiguous. Requirements elicitation is challenging for an analyst as this phase of requirements engineering (RE) explores the boundaries of knowledge, the people who possess this knowledge and how to acquire that knowledge [3]. In RE, interviews have been the most widely used elicitation technique, and are considered among the most effective in terms of data collection $[4,5]$.
In RE Education and Training (REET), the effectiveness of analysts in conducting requirements elicitation interviews highly depends on having experienced and actively participated in real interviews [6]. However, empirical evidence has shown that the methodological soundness and correct conduct of interviews is also important [4]. Therefore, in principle, both novice and experienced analysts can elicit high-quality requirements when the interview is well-planned. Mistakes made during design and execution of the interview task can impact the resulting software and system requirements [7]. An important part of training students on how to plan and perform elicitation interviews, is to teach them how to prepare for the interview (e.g. by composing the right questions, making rapport with the interviewee, etc.). Another essential element of training is to bring awareness about the mistakes often made by novice analysts during these interviews. Students can learn from their mistakes based on the feedback provided by the trainers and improve their skills by practice. Feedback based pedagogical approaches have been applied effectively in various other disciplines for teaching $[8,9]$.

Research has demonstrated the effectiveness of role-playing pedagogical approaches in REET by providing authentic assessment for the students $[10,11]$. The educational approaches designed with authentic assessment require the educator to simulate the real world environment aimed at student learning by practice [12]. Mistakes, if observed explicitly during practice (even in simulation), can become a learning resource for students in the form of feedback. Furthermore, a comprehensive catalogue of mistakes made during elicitation interviews can be utilized in REET courses to help students better prepare for their role-playing activities.

In this paper we present the results from our empirical study that aimed to identify the mistakes made by student analysts during their role playing in their first requirements elicitation interviews. Participants of this study were 110 post graduate students in University of Technology Sydney teamed up in 28 groups to conduct requirements elicitation interviews with a business owner (role played by an experienced academic). To identify the mistakes, the interviews were audio recorded and the recordings were reviewed by two experienced researchers. 
Furthermore, observation notes were also taken by another researcher during the interviews. Reviews and observation notes were qualitatively analyzed to identify the themes and to classify all the mistakes. Our empirical study presented in this paper builds upon REET body of knowledge in general, and the preliminary work of Donati et al [6], in particular. Our main contribution is the identification of list of 34 unique mistakes made in elicitation interviews that are classified into 7 high level themes. We also give examples of the mistakes made by the novices, to provide contextual information and indicative recommendation, which can assist the educationists and trainers for teaching the art of elicitation interviews. Our rigorous research design is a novel combination of several well-known pedagogical approaches that we used to conduct this observational study. It has been described in sufficient details to make it repeatable for future REET research.

The paper is organized as following: section II summarizes the background and related research work available on interviews. Section III highlights our motivation for the research. Section IV gives details of the steps of research methodology and section V describes the results. Section VI discusses the implications of the research. Section VII provides conclusion and future directions.

\section{BACKGROUND AND RELATED WORK}

Requirements elicitation interviews are recognized as one of the most effective and used technique to elicit requirements [4]. Nevertheless, only a little part of the RE community's effort has been focused on studying interviews and, more in general, elicitation techniques [13].

Most of the existing work on interviews focuses on identifying the variables that affect the success of an interview. In particular, the influence of domain knowledge [14-17], and cognitive strategies [18] were evaluated, as well as the combination of other individual factors, such as the expressive ability of the customer, and the absorptive capacity of the analyst. In [19], the variables that affect interviews have been categorized in three main classes: human-oriented, process-oriented, and contextoriented factors. For some of the analyzed factors, both positive impact and a negative impact have been identified. Two examples of these factors are: 1) domain knowledge [15], which, on one side, can help to prepare better questions and use a more appropriate language, and, on the other, might convince the analyst that she knows the answers better than the customer; 2) ambiguity [20], which is usually perceived as an obstacle to knowledge transfer, but, once identified in interviews, can lead to disclose tacit knowledge.

Another relevant factor for the success of interviews is the adequacy of communication. In this context, through a theoretical study, Coughlan and Macredie [21] identified articulation, misunderstanding, and conflict as the general classes of problems that hamper communication during requirements elicitation. Through empirical studies, instead, in [22, 23], possible structures and models for the communication during the interviews have been identified with the goal of improving the effectiveness in collecting requirements. Other works did a step forward and look at how to improve communication in interviews through precise guidelines. For example, Pitts and Browne [7] showed that using procedural prompts that stimulate cognition, instead of interrogatories ones, lead to more successful interviews. Shuraida and Barki [24] showed that analysts who encourage the use of concrete examples are more likely to produce satisfactory requirements. From a practitioner's perspective, Portugal's work [25] provides a large set of guidelines, based on the author's experience, to conduct a successful interview. None of these works, however, provides a set of guidelines of what to do and not to do in a requirements elicitation interview, or a comprehensive analysis of the most common mistakes of analysts, especially the young ones, who lacks of experience and of the needed skills, which, together with communication talent, are one of the factors that mostly affect the quality of interviews $[2,15]$. An initial work in this direction is [6], where Donati et al. identified and catalogued mistakes that student analysts commonly do in interviews. The mistakes were derived from a thorough analysis of a set of student-performed interviews.

Besides this initial work, the literature does not offer any tool to effectively teach how to perform a successful interviews, which should be one of the primary objectives of requirements engineering courses [11]. Unfortunately, because of the lack of tools and the lack of time, however, this objective is often neglected. Indeed, computer science related degrees either offer only a course on requirements engineering, which should include all the different activities related to the discipline or, even worse, offer only a software engineering course in which requirements engineering is relegated to a couple of lectures.

Given the lack of preparation of many analysts and the importance of this activity, many online trainings and courses have been created to help analysts to conduct more effective interviews. Lynda.com [26] offers a one hour and a half subscription training composed by 5 modules in which the main aspects of an interview are covered. The course also contains examples and challenges. Interviews are also taught as first-class citizen in subscription specialization online courses (e.g., $[27,28])$ in which video-scenario are provided to better contextualize the taught concepts. A training for interviews and workshops for IT projects is provided in a book form in [29]; this training includes initial definitions, motivations, and some guidelines. Also, short YouTube videos are provided to identify the main characteristics of requirements elicitation interviews and the most needed skills to succeed in them. However, none of these trainings and video deepens in the analysis of the communication problems, systematically analyze all the most recurrent mistakes, and proposes solution for them. Most of them are only mainly based on the experience of the training developers.

Besides RE, interviews are important tools also in other disciplines, such as journalism, psychology, qualitative research methods, and criminal justice. In these fields, the analysis of interviews and the tools provided to teach them are in a much more mature state and have been developed through thorough research and deep analysis and experience. A large body of literature is available on how to conduct interviews in these fields 
and which common mistakes to avoid. In journalism, for examples, books such as [30], provide a practical, well-structured, easy-reference guide for journalists at any entry level: students, trainees and novices. It covers the analysis of interviewing techniques, the types of interviewees and how to read body-language. Since interviewing methods can differ depending on the goal of the interview, there are books specific to different products, such as [31], in which Martin discusses interviewing methods for actuality documentaries, deeply analysing how they need to be run and prepared, and which situations to avoid. The field offers also books by the most expert journalists, such as [32], in which the readers can learn from the authors memorable experiences and analyzes of them. It has to be noticed that journalism is an independent academic discipline with an autonomous degree [33] and this explains the abundance of material that targets young interviewers.

In social sciences, such as psychology, interviews are used as a double instrument, to collect qualitative data for research or to interact with patients. People interested in using them to collect data for research can refer to an extensive literature, that comprise both introductory works that define the different types of interviews and data collection methods (e.g., [34, 35]), more practical works that provides tips for running interviews (e.g., [36-38]), and books that generally contain both (e.g., [39, 40]). The tips-focused papers target either students [36] or inexperienced analysts [37, 38]. Among the other tips, Jacob and Furgerson [36] encourage students to go into an interview with a script that covers it from the beginning to the end. The script should consist of the reasoning behind the interview, explanations as to how the interview should progress, and a little introduction to build rapport between the interviewee and the analyst. This does not necessarily mean that the analyst cannot deviate from the script. In fact, it is encouraged that the student be willing to make 'on the spot' revisions to the interview protocol. The script should be used to guide the interview process, so details that need to be questioned or mentioned do not get missed in the conversation between the interviewer and the interviewee. This is in line with Diley's suggestion of working on an accurate protocol before walking in an interview [38].

On the practitioners' perspective, psychology, being as journalism taught as a university major, includes precise guidelines and provides tools for students and young practitioner to correctly run interviews. A comprehensive example of these guidelines is provided in [41], which is a manual on interviewing mental health patients based on objective research and bestpractice principles. Other works in the field focus on giving recommendations, such as focusing on positive aspects while interviewing [42], or analysing strategies depending on the considered mental disease [43]. Interview techniques and skills are deeply studied also in criminal justice, where interviews are distinguished from interrogations, legal issues are faced, and different criteria are applied [44]. Besides traditional tools, trainings [45] are also available to cover the theory behind interviews and to practice through role-playing exercises.

The professionalism and quality of the results in conducting interviews in the above-mentioned fields suggest the need of producing similar guidelines, based on research, also in requirements engineering. Unfortunately, given the differences in goals and in the relationships with the interviewees with respect to these disciplines, new studies to deduce field-related guidelines are needed.

\section{MOTIVATION}

The authors of this paper belong to four different academic institutions in Europe, United States and Australia. Our experience of teaching RE courses in the last 2 decades both at undergraduate and postgraduate level has provided a rich tapestry of issues and challenges for REET. We have experimented with utilizing several pedagogical approaches to enhance and improve the learning outcomes. Our interest in conducting the study reported in this paper was not only inspired by our teaching experiences but also partly triggered by a similar study by Donati et al in REFSQ 2017 [6]. Our study differs fundamentally from their work in a number of different ways that we describe below. Many of these differences are informed by our previous experiences of teaching RE classes using role playing, as well as several self-identified limitations and some of the deficiencies we observed in [6]:

- Participants - In our study we had 110 first year Master of IT students engaged in elicitation interviews as part of their first assessment task in their RE class. Donati et al, engaged 38 undergraduate students in their $3^{\text {rd }}$ and $4^{\text {th }}$ year in their "User Centered Design" course.

- Role playing - In our study the role of customer was played by an experienced RE researcher and instructor who was also the tutor for this course; while in their case, half of the class played the role of customer and the other half the role of analyst. The decision for not using students to play customer role was based on the results of $[10,11]$. So, in our study, we had a single customer who was able to do consistent delivery of responses to questions in the interviews.

- Case studies - In the study by Donati et al, the customer participants were required to think about a "novel computer intensive system" for interviews, while our participants were divided into two and each half was given a different case study prepared by the instructor in the form of a onepage project brief to commence.

- Preparation - Donati et al prepared the analysts by two hour lecture on requirements elicitation interviews. Our participants were told to do the short course on requirements elicitation interviews on Lynda.com. They also attended an introductory lecture on requirements elicitation and more specifically on how to plan and prepare for interviews. In this lecture and the follow up tutorial, students were exposed to a number of common mistakes students make in their interview that included the list from Donati et al. Finally, we designed and created a few video recordings of good and bad interviews that was made available to students to help them in preparing for interviews.

- Conduct of the Interviews - Our interviews were semistructured while theirs were unstructured. Their interviews were one on one, whereas our activity was designed for 
collaborative learning, hence a team of 3 or 4 group conducted the interview with one customer.

- Interview output - There was no written output required from the analysts after the interviews in Donati et al's study. In our study all the groups were asked to submit minutes of their interview for assessment.

The remainder of the differences between our observational study and Donati et al's work will be revealed in the next section.

\section{RESEARCH METHOD}

\section{A. Study context}

Our motivation for this study comes from many years of academic experience of observing how university students (both undergraduate and post graduate levels), struggle to learn effective requirements elicitation interviewing techniques. Over the years, we have attempted to inform students on an ad hoc basis about possible mistakes that one can make in interviews. To do this task more efficiently, we have recognized a need to have an empirically validated list of possible mistakes and the corresponding examples to provide to students in a more formal manner. Therefore, the overall aim of this observational study is to develop such a list and related examples to assist students in learning the skills of effective elicitation interviews.

\section{B. Study setting and participants}

The study was conducted in a university setting with master level students of Information Technology enrolled in "Enterprise Business Requirements" class. The first assessment task was for students to develop a complete software requirements specification (SRS) for a customer based on a one-page project brief provided by the instructor (second author). The 110 students were grouped into 28 teams comprising of 3 to 4 members. Each team was instructed to conduct three interviews over three weeks with the customer, to elicit the requirements. After the completion of each interview, students were instructed to submit the minutes of their meeting with the customer on a specially designed template within 2 days after the interview to capture what they have understood. It should be noted that the observations and analysis presented in this paper are entirely based only on the first interview. The first part of the deliverable was a set of use cases developed from the information elicited in the three interviews. The final part of the assessment was a complete SRS using the IEEE standard template. The requirements elicitation interviews took place after students attended lectures on requirements elicitation and relevant techniques, attended a workshop for practicing interviews with customers and were also asked to watch the video courses on Lynda.com about "Requirements Elicitation for Business Analysts: Interviews" [26] and do the exercises given. Two case studies were designed by the instructor and the class was divided into two, one half did the first case study while the other half tackled the second case study. Students were provided with the vision statements of the case studies for which they had to prepare an interview. The one page vision statement briefly described the current business process and the need for a new system. All groups were allocated 15 minutes for each of the three interviews with the customer of the case study they were assigned. We adopted the corrective feedback learning approach for the whole task. The aim of observing mistakes was not to assess the students for the quality of the interviews but to provide them feedback for the next round so they can improve their interview skills. The interviews were conducted as a role-playing activity with authentic assessment pedagogical setting $[10,11]$, in which we simulated a real world environment for the students to perform interviews with a customer. The task was collaborative in nature. The students were expected to plan for the interview as a group for various tasks divided among members, such as preparing questions, asking questions, taking notes, audio recording interviews, preparing minutes of meeting.

\section{C.Data Collection and Analysis}

The research is exploratory and interpretive in nature and we used a qualitative approach to data collection and analysis. We had multiple Requirements Analysts; RAs (28 groups of students), a Customer; C (role played by the first author for all the groups, an academic and experienced RE researcher), an Observer; O (Third author, experienced RE researcher), and two Reviewers; R1 (Lecturer from another university; the fourth author), and R2 (a Business Analysts; the fifth author). The data were collected in three ways: audio recordings of the interviews, about 7 hours; the observation notes of the researcher (O), 4451 words; think aloud of the customer after every interview (C) in conversation with $\mathrm{O}$ (who took notes), 1635 words. The audio recordings were reviewed by two Researchers (R1, R2), and qualitatively analyzed independently for the mistakes the student analysts made in each interview, producing 4748 (R1) and 3546 words (R2). The use of "think aloud" was oriented to identify the mistakes perceived by the customer's role during the interview, which may be different, also in terms of perceived relevance (e.g., rapport with the customer), from those that could be observed externally. Overall, a total of 14,380 words (about 32 pages) of data was produced for further analysis. We had additional 28 documents of minutes of meetings submitted by all the groups after the interview. Figure 1 presents the overall method of data collection and analysis.

\begin{tabular}{|c|c|c|c|}
\hline Data Collection & Data Review & Data Analysis & Results \\
\hline Audio Recordings & Review by R1 & \multirow{3}{*}{$\begin{array}{c}\text { Thematic Analysis } \\
\text { and Synthesis }\end{array}$} & \multirow{3}{*}{$\begin{array}{l}\text { List of } \\
\text { interview } \\
\text { mistakes } \\
\text { classified } \\
\text { into themes }\end{array}$} \\
\hline Observations by & Review by R2 & & \\
\hline $\begin{array}{l}\text { Customer think } \\
\text { aloud (C) }\end{array}$ & & & \\
\hline
\end{tabular}

Fig. 1. Steps of data collection and analysis

Two of the researchers (First and Second authors), carried out the thematic analysis of all the data and synthesized the list of mistakes into classified themes. The four sources of data (two reviews, observation notes, and customer think aloud) were first stratified for individual groups for further analysis. Some mistakes were observed in all four sources of data, whereas there were cases of additional new and unique mistakes identified from the two reviews based on audio recordings. Our findings concur with $[46,47]$ that review of interview audio recordings 
provides more insights and reduces the bias of observations by triangulating the data from neutral perspective, as the reviewers are not being present at the time of interview. All the recorded mistakes were coded to identify the unique mistakes for each group and later analyzed for their frequency of occurrence in all groups. The mistakes were further classified into higher level themes corresponding to the particular aspect of the interview. The final classified list of mistakes was peer reviewed by one researcher (third author). There were instances of disagreements related to the naming of themes and grouping, and they were resolved in discussion. After synthesizing and categorizing the list of mistakes, we reviewed the minutes of meetings submitted by all the groups. The aim was to investigate any plausible relationships between the types of mistake made during interviews and the extent of students' understanding based on what was recorded in the minutes. For this purpose, we had to go back and listen to some of the audio recordings again for further analysis.

\section{RESUlTS}

In this section we present the results from our analysis and discuss the findings.

\section{A. Interview Mistakes}

We identified 34 unique mistakes classified into 7 higherlevel categories of mistakes:

1. Question Formulation

2. Question Omission

3. Order of Interview Questions

4. Communication Skills

5. Analyst Behaviour

6. Customer Interaction

7. Teamwork and Planning

Figure 2 shows the list of classified mistakes along with their frequency of occurrence observed in 28 groups. The most frequently observed mistakes are (1) asking vague questions, (2) incorrectly ending of interview and (3) not building rapport with the customer. We discuss these categories in the following by providing examples from our qualitative data. Some of the examples may demonstrate more than one type of mistakes.

\section{1) Question Formulation}

This category of mistakes refers to the problems and issues about the questions that student analysts asked the customer during their interviews. In a well-planned interview, the analysts have time in advance to prepare for writing down clear and unambiguous questions [6]. A response to the question depends on how the question is formulated. Vague, incorrect or unclear questions are rarely going to elicit correct responses from the customer.

The major mistakes observed in this category are: (1) asking vague questions, (2) asking technical questions, (3) asking irrelevant or incorrect questions, and (4) asking customer for solutions. We now give examples of the excerpts from the data used in the study. Some of these excerpts may also include the exact questions asked or statements made by the students in the interviews.

\section{a) Asking vague questions}

'Asking vague questions' was the most frequent mistake made by student analysts and was observed in 21 instances out of 28 interviews. We define vague questions in this context as the type of questions that may yield multiple interpretations, or cases where no reasonable meaning can be inferred from the questions asked. The ambiguities that can result from the response of the customer to a vague question can create further issues in later stages [48]. We provide some of the indicative examples of 'vagueness' in interview questions from the reviews and observations. The reviewers observed that the student analyst asked vague questions on success, failure and risks of the project.

- "The [analyst] asks about measuring success, the question is always vague ..., and [the analyst] does not propose a way to measure success. They ask about risks, but the [customer] doesn't know how to estimate"

- $\quad$ "Some questions are not understood by the customer because they are too vague or posed at the wrong time ... [they]. are out of context at the end of the interview when goals, success criteria and motivations have already been discussed"

The following were the examples of the questions asked by students that were pointed out by the reviewers to be "too vague" for the customer, and were asked out of context, therefore the customer could not provide appropriate response:

- "What is the impact of the project to your business?"

- "Can you indicate the major constraints of the project?"

- "Do you want some specific features on the website?"

- "What are your expectations?"

Asking these types of questions are hardly going to trigger the reasoning or stimulate follow-up discussion with the customer [23] and they indicate that the analyst is inexperienced in the art of question formulation [49].

\section{b) Asking technical questions}

Our data was collected from the first interview with a customer, who is in fact the project sponsor and business owner. The students were expected to have researched the customers' business context and prepare appropriate questions. Asking 'technical questions' from the customer so early may not get an adequate response because it cannot be assumed that the business owner/project sponsor has detailed technical knowledge. Asking technical questions may also intimidate the customer and can lead to bad rapport. Following are the excerpts of the reviews on asking technical questions:

- "Often the interviewer assumes that the stakeholder has a technical background: questions on "secure" or the use of Oracle. [The analyst] never checks on the common vocabulary with the interviewee and is not concerned about some possible previously happened misunderstanding even when the [customer] tells [the analyst] that [the customer] does not have a technical background."

- "part of the interview is devoted to purely technical aspects that perhaps may be left to a second step [next interview]" 


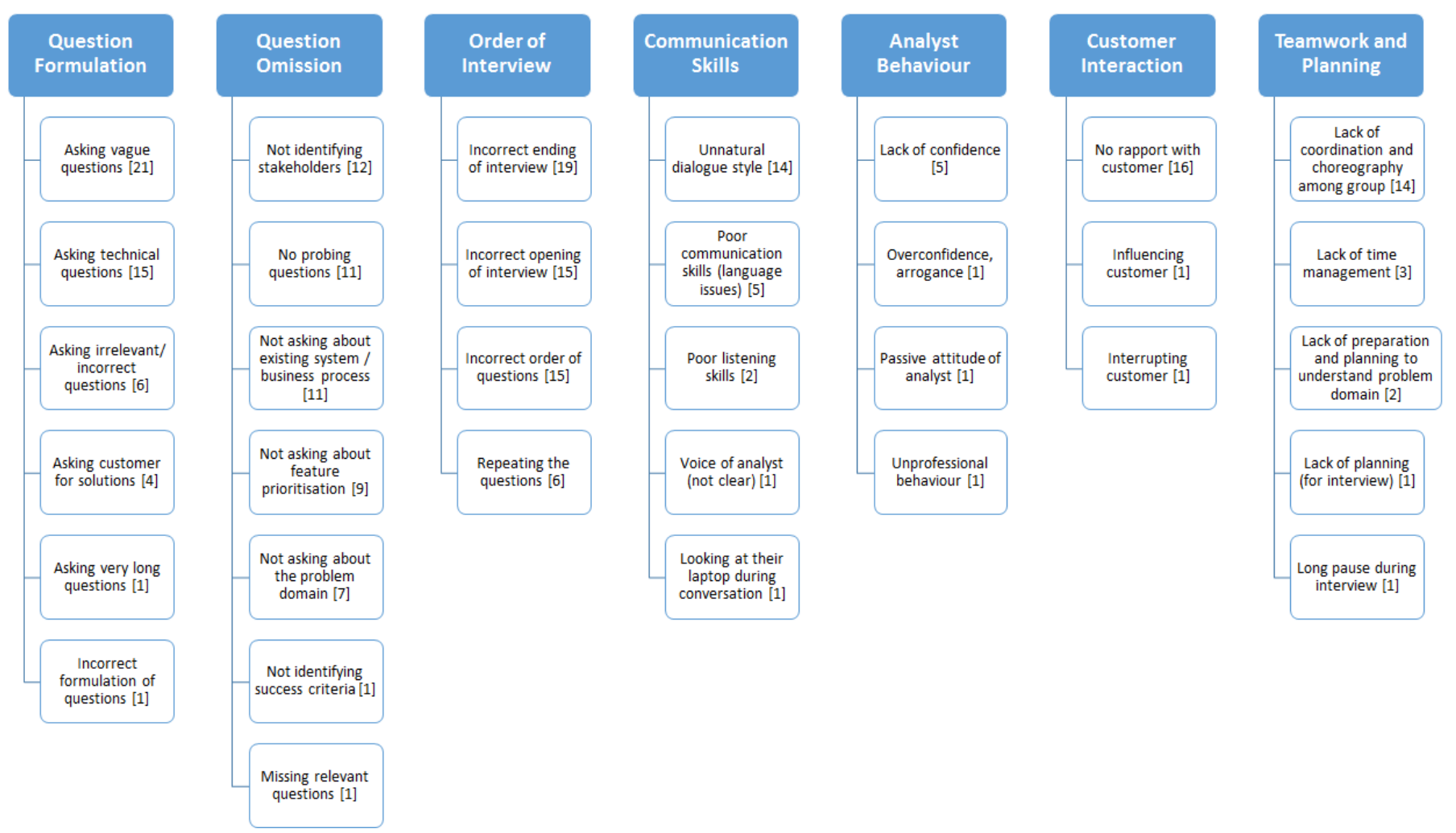

Fig. 2. Classification and frequency of interview mistakes

The reviewers provided examples in some cases about the questions that students asked:

- "The analyst uses often technical language: 'How do you map the business goals to the system goals?', or again 'What is the minimum viable product'(!), the customer can't understand and asks for clarifications,"

- " "If the system fails do you have a backup?"; the customer doesn't understand this question because [she] is not the right person to be questioned about technical features."

\section{c) Asking irrelevant or incorrect questions}

This category refers to asking questions that are not relevant for the development of the system, or are inappropriate for the profile of the customer. Asking 'irrelevant or incorrect questions' will not only waste the time during interview session but also will add to the irrelevant data elicited during interview that might contribute to creating erroneous or redundant requirements. Asking these types of questions has been recognized as one of fundamental mistakes in requirements elicitations [50].

As the reviewer observed in one example case: "They asked an incorrect question, concerning the customers having access to the inventory". The question was asked even though the customer had explicitly mentioned the role of a person who deals with the inventory. Another example observed by reviewer was the way the student analyst explained the security of the system - "The security question scares the customer" and persuaded to express concerns on the security that was not required.
The other less frequent mistakes observed in this category were the students asked customer for "solutions", or asked a "very long question" that the customer has to ask for repeating or rephrase multiple times.

\section{2) Question Omission}

This category of mistakes refers to omitted questions that were expected to be asked by the student in the first interview. In this category the most frequent mistakes observed are when student analysts (1) did not ask to identify relevant stakeholders, (2) did not ask follow-up or probing questions, (3) did not inquire about existing system or business process, (4) did not ask the customer to prioritize the features and (5) did not ask about the problem domain. Missing these types of questions could potentially lead to missing requirements in later stages.

Below are examples of excerpts from comments identified in our data:

- "[analyst] did not ask about stakeholders, they did not look very well planned."

- "they [analysts] did not ask probing questions ..., like they thought that the maximum information that could be elicited was reached already."

- "They [analysts] did not ask about the problem or the existing system/process. Overall, they have details, but not the [bigger] picture, while this should be made explicit in the interview."

- "They [analysts] did not prioritize the features that were required by the customers."

- "They [analysts] could not provide examples ... when [the customer] asks to elaborate, they couldn't. They appear 
not having thought about the domain ... questions are all generic, domain independent."

This exercise was the first stakeholder interview for the student analysts, and they were expected to find out the relevant people in the business and decide who they would interview next. Stakeholder identification is one of the important activity in requirements elicitation [51]. 12 out of 28 groups did not identify relevant stakeholders. A possible explanation for this mistake, as pointed out by [51], is that analysts mostly view stakeholder identification as a 'self-evident task', or they attempt to have less conflict of interests arising from the point of view of different stakeholders. Not asking probing and follow-up questions during the interview would fail the purpose of face-to-face communication, as interviews are reported to help analysts resolve the ambiguity that emerges during the interview [20], and to push the customer to express the tacit knowledge about the existing business process or system [52].

\section{3) Order of Interview Questions}

This theme refers to the mistakes about the overall order in which the questions were asked, i.e. the start of the interview, the order in which the questions are asked, and the ending of the interview. The order in which the questions are asked creates a flow of conversation that should lead in a logical way for customers to explain the project vision, and explain why they need a system within the existing business process. It was frequently observed that the student analysts did not make an attempt to have a good start and/or end to the interview but also asked the questions in incorrect logical order throughout the interview.

The excerpts from the data showed multiple examples in which the students did not try to build rapport with the customer at the outset, they asked questions about solutions before understanding the problem, and ended the interview abruptly without any final summary of the collected information.

- "They [analysts] do not introduce each other and asked suddenly 'what is the feature of success?'. This question should be asked later."

- "the interview begins with a series of direct questions even if we would expect a general description of the project."

- "the dialogue ends abruptly and a final summary is missing"

- $\quad$ "The questions are also in the wrong order, for example the very first one is 'What are the project's success criteria for you?' and only after he [analyst] asks 'What do you want to get?' and after that he asks 'What are the goals? " It is necessary for the analysts to form a questioning strategy and include prompts based on the context of their interview, this can help in eliciting particular requirements as well as overcoming the challenge of customer-analyst interaction [23]. Prompting strategies can provide an opportunity for the analyst and user to re-evaluate acquired information. This should result in a more complete and more accurate set of requirements [7]. The recommended strategy to the student in this exercise was to: start the interview by building rapport with the customer, understand the existing business process, understand the problems faced by the customers in current process in order to reason on the need for a new system. Towards the end of the interview, summarize the findings to the customer to confirm the understanding. 19 out of 28 groups did not summarize the findings, and 15 groups did not open the interview correctly and asked questions in the wrong order. Summarizing the findings of the interview is a best practice for overcoming the misinterpretations during the interview [7] and overcoming any cognitive limitations during customer and analyst communication [23].

\section{4) Communication Skills}

Interviews are a communicative intensive activity in which the analyst has to be involved in a face-to-face communication with people from diverse backgrounds, skills and knowledge levels [21]. In order to create a shared understanding with the customer during the interview, the communication skills of analysts are crucial. The analyst has to work on extra effort to remove the semantic gap and push the customer to the boundaries of their tacit knowledge. Effective communication has always been quite challenging for the analysts who are dealing with customers, and is one of the most recurring issues in requirements elicitation [53]. The data collected in our study in many cases pointed out that the dialogue with the customer was not considered as a natural conversation but more of a rehearsed sequence of asking questions like interrogation. This can make the customer uneasy. The use of common vocabulary during interview is also very important and the analysts should plan and prepare so that they will not use the words that might confuse the customer. The following examples from review excerpts demonstrate the observation made about the poor communication skills of student analysts:

- "The dialogue is confusing and customer doesn't understand the questions, mostly due to poor linguistic skills (of the analysts)."

- " $\quad$ the fact that the [analysts] are clearly following a series of good practices they read on a book make the structure of the interview rather scholastic and the dialogue unconvincing in some points. I think maybe some deviations from the 'script', based on more personal insights, might have led to discovering other mid-level details of the project that are left aside and could help the developing process."

- " the main weaknesses of this interview are due to the passive attitude and poor dialogic skills of the [analyst]. He often express himself in ambiguous terms and this make the dialogue ineffective. The fact that the client often doesn't understand the questions is strongly negative."

- " "the customer feels that the analyst is not listening, ... the analyst is reading the paper."

- "They [analysts] are not listening, so they keep making the same mistakes and they sometime ask the same question twice."

The difference between interviews and a survey questionnaire is that the former technique offers analysts the opportunity to have a face-to-face interaction to build an understanding with the customer by asking further questions based on the previous responses. But if the analyst is not carefully listening to the customer, or interrupts them in the middle of a response, or asks interrogatory questions, the benefits of face-to-face interviews get lost [6]. 


\section{5) Analyst Behaviour}

The behavior of analysts during interviews can impact on the attitude of the customers and influence their responses. Specially the overconfidence of the analyst can potentially lead to incorrect understanding of the problem domain and would prevent the analyst to look for alternative or contradictory information [7].

Although we did not come across too many observations regarding the behavioral aspect of student analysts, following are a few examples that were classified into this category.

- "the ones [analysts] with professional experience looked overconfident, they thought they did not make mistakes, but they were totally out of what was asked in the assignment, and they looked like they invented the interview questions in the moment"

- "[analyst] looked too much in a hurry, talking too much, and had an aggressive start, he did not introduce himself, and the others."

- "... problem is the unprofessional attitude of students who often laugh and go too fast as they are embarrassed."

- "the low voice and the slow attitude are really hard to tolerate."

- "the analysts seems a bit nervous. They do not introduce themselves."

\section{6) Customer Interaction}

As asserted previously, the successful outcomes of an interview activity relies heavily on the analyst-customer interaction [54]. It is typically the responsibility of the analyst to create a friendly environment that can stimulate the communication with customer $[55,56]$.

'Not to build rapport with the customer' at the outset of the interview was the third most observed mistake. 16 out of 28 groups made this mistake with the student analysts starting to ask direct questions from the customer straight away. This behavior can intimidate the customer and can create an uneasy environment for the customer to properly express their ideas and vision to the analysts. Following are some of the examples from the excerpts of the interview reviews identifying mistakes of 'customer interaction':

1. "The [analysts] do not introduce themselves, moreover, even if they ask initial ice breaking questions, it sound more as an exercise and the speaker does not really sound interested in the answers."

2. "[analyst] interrupted before the customer could complete the discourse."

3. "They [analysts] did not create rapport, and did not ask who to talk next."

4. "They open [interview] without building rapport"

5. "Shaky start, he [analyst] looked not convinced in asking questions, he did not looked confident ... building the rapport looked a bit fake"

6. "In addition they [analysts] spent too much time trying to promote their ideas even when the customer doesn't agree."

\section{7) Teamwork and Planning}

In the context of this study, interviews were conducted as a group task, and there were instances in which the lack of planning and coordination among team members was easily observed. In some cases, the team did not have a planned choreography of task divisions for asking questions and taking notes, and the interviewers would interrupt each other. In other cases, they did not profit from the 15 minutes allocated for the task, and they either made long pauses, or ended the interview earlier. Excerpts that represent the different mistakes observed in this group are reported below.

- "if two people are confident, it does not work, because they interrupt each other, because they feel like being in action, and this does not appear productive for the success of the interview"

- "lack of coordination with additional people arriving [late]"

- "they did not look like a group, there was no coordination ... they also did not build any rapport, making the customer defensive since the first meeting."

- "There was also an apparent lack of planning both for the objective of getting information (in terms of sequence of questions to ask), and for the organization of the interaction during the interview (in terms of who will speak)."

\section{B. Impact on the quality of elicited information}

Mistakes made during design and execution of the interview task can impact on the resulting software and system requirements [56]. The minutes of meetings in this activity were used as a tool for assessment of the student analysts' comprehension of the responses given by the customer. We further reviewed and analyzed the minutes of meetings submitted by the students recorded immediately after their interview. In our review of the sample of minutes of the meetings, we traced the types of mistakes identified during our thematic analysis to what was recorded in the minutes - the minutes recorded both the original questions asked, and the responses of the customer. Although we cannot claim a direct causal relationship between specific mistakes made in the interview to what was recorded in the minutes, however, a general pattern was observed that the groups who made mistakes (specifically in question formulation and question omission), have articulated their understanding poorly in the minutes. We offer a few examples of this phenomenon from our analysis.

- In one case, the reviewers reported that the group was "asking vague questions", and we extracted several vague questions recorded from their minutes. For example this question was recorded in the minutes: "what do you think is the better performance". The response to this question was recorded as: "The owner hopes the new system can support online operations for customers, such as request tracking". This shows that the vague question resulted in a vague response recorded, which has nothing to do with performance and reflects the poor understanding of the students.

- In another instance, the reviewers reported that the group was "asking irrelevant questions", we observed this question in the minutes of meeting: "How do you have an understanding of your company daily operations such as the 
number of the customer and the services applied per week?". The recorded response was: "It depends, every week it's different. they cannot predict in advance how many people per week, If we want to know exactly, we can ask one of [the] senior employees". This was perceived to be an irrelevant question for the first customer interview and from the response it appears that no relevant information was elicited.

- Another example is related to the mistake named "asking technical questions". In one case in the minutes the recorded question was: "Why do you think an Internet-based service system could be useful for this project?". The response recorded to this question was: "Call system is problematic about tracking service staff so if it's online it will help it tracking staff and their availability which will streamline business process and save time as well". The response indicates that the customer didn't really understand the technical question. Once again, no useful requirements information was elicited by this question.

- In all the instances where the reviewers reported the "Question Omission" category of mistakes, we checked the minutes of the meetings to verify that indeed those questions that were expected to be asked were not recorded in the minutes.

\section{DISCUSSION}

In this paper we have presented the results from our observational study of analyzing the mistakes made by novice analysts (students) during their first interview with the customer. In this section, we compare and contrast our findings with previous relevant research.

\section{A. Domain knowledge}

Effective requirements elicitation largely depends on the familiarity of the analyst with the problem domain $[15,16]$. In our study, the students were provided project briefs for their case studies in order to do their research and planning for the interview. Requirement analysts can be more effective if they have developed a good understanding of the problem domain [14]. The domain knowledge helps the analyst in proper planning of the interview, in developing shared understanding with the customer, and gathering and organizing the acquired information into complete and clear requirements [7]. Looking at the mistakes observed, we argue that the lack of domain knowledge by the student analysts potentially contributed to several of the mistakes made in question formulation or omissions. For example in our category of question formulation, the mistakes observed regarding the students asking incorrect or irrelevant questions (Figure 2) were mostly due to lack of understanding of the problem domain. A well planned interview can address this issue.

\section{B. Minutes of Interviews}

For instructors, it is important to assess the level and extent of students' ability to perform an effective interview. In order to achieve this goal, we asked students to write minutes of their interview immediately after the completion. The content of the minutes gives us a good indication of the level of students' understanding of the application domain and the initial requirements developed in their interview. In this study, we observed that the students who performed well during their interview (based on the assessment of the customer articulated in the think aloud), also produced reasonably good quality minutes. However, there were also a few cases where the minutes were of poor quality even though the interview was assessed to be reasonably good. So, it is not just enough to ask the right questions in the interview, but it is also equally important to listen carefully to the responses given by the customer and accurately record the understanding developed. This may be due to the fact that many groups did not present a summary of the interview discussion to the customer at the end of the interview (i.e. incorrect ending of interview).

\section{Rapport with Customer}

Our results revealed 16 cases of analysts not attempting to build rapport with the customer. Many of the cases where attempt was made to do so, seemed rather unnatural and essentially copied the utterances given in the Lynda.com online course that students accessed to prepare for the interviews. We assert that teaching students this particular skill is rather challenging as it does not come naturally to many. We recommend that students rehearse their interviews, and if possible, record it and try to improve it with practice before coming to the real interview. However, this is a skill that would ultimately only improve with practice in real settings.

\section{Ambiguity as a resource}

The results have revealed that 21 out of 28 groups have made the mistakes of asking vague questions. We observed that the responses to those questions in the minutes were also ambiguous. Since the design of our assessments were developed following the corrective feedback learning paradigm, we adopted the idea presented in [20] to use the ambiguity in the interviews as a learning resource for students in preparing follow-up questions for the next round of interviews. Although our study in this paper only focuses on the observations made during first interview, we asked the students to identify the ambiguous responses in their minutes to formulate questions for the next round of interviews. Our observations of the questions asked in the follow up interviews (based on the minutes of meetings of second and third interviews), reaffirmed our intuition that this approach was very effective, which resulted in students improving their understanding of the requirements. We recommend this resource to all instructors as an effective teaching tool.

\section{E. Experience versus planning}

The systematic review of Davis et al [4] has revealed that a novice analyst, with careful planning for the interview, can elicit information equally as well as an experience one. In our study, one of the students already had experience of being business analyst and relied on his experience during the experiment rather than planning and coordinating with the group. That group made more mistakes due to his overconfidence and intimidating behavior towards the customer. Giving customer an impression that the IT people 
know it all is a bad practice and impedes the formation of trusting relationship between the two sides which should be a critical aspect of the first interview.

\section{F. Contribution to the Body of Knowledge}

Our observational study reported in this paper has generated new insights both in REET research as well as into the development of new research design for education. We have developed a systematic empirical approach to study the mistakes that novice analysts make in their first encounter with a customer during elicitation interviews. Our research design is substantially more rigorous that the only study of this kind previously published in RE [6]. Furthermore, by applying this research design in the curriculum development and performing the observational study we have extended the number of the previously identified mistakes [6]. These new mistakes relate to group behavior and organization as well as attitude of the novice analysts. We have also presented the frequency of the mistakes since our sample was substantially larger. Besides the contribution of our study to the Body of REET Knowledge, we believe the findings are important to educationists and trainers in the following ways:

- We reaffirm that role playing $[10,11]$, is a very effective method of training in REET, in particular, requirements elicitation interviews.

- We have presented a curriculum design that utilizes a collaborative learning environment which is considered as an effective pedagogical approach in RE.

\section{THREATS TO VALIDITY}

We have conducted our thematic analysis under interpretivist paradigm [57], which relies on the interpretation of the construct through the understanding of the researcher. In regards to the qualitative analysis in this paradigm of inquiry, it is impossible to claim absolute exactness of the results free from researchers' bias. The researchers are expected to draw on their knowledge to produce insights from observations and build concepts from which their theory emerges. However, we have tried to mitigate the risk of researcher's bias and increase the reliability of the results in our research design through independent reviews of researchers who had nothing to do with the course delivery and two of them were not even present during the interviews. One of the reviewers is an instructor in another university and one is a BA practitioner. With this in mind, we consider that we have provided sufficient details of the process of data collection and analysis in this paper to indicate the reliability and increase the trustworthiness of the results. R1, R2, O and $\mathrm{C}$ may have been biased by the knowledge of Donati et al, when looking for mistakes. Although we explicitly asked them to do their analysis without considering this previous work, this threat could not be entirely mitigated. However, the identification of 21 additional mistakes with respect to Donati et al., shows that this threat was addressed in practice. The multiple role of customer, instructor and researcher of the first author is also a source of bias in the data analysis phase. This is mitigated by the presence of different, independent viewpoints in the different phases of the data collection and analysis process. The behaviour of the students, and therefore the commission of certain mistakes, may be influenced by the relation of the students with the instructor, who was playing the role of customer. Different behaviours may be observed with real customers. Although this threat could not be fully mitigated, it should be noticed that the instructor had previous experience in role-playing, and this allowed her to play the customer's part with sufficient realism. This allowed a partial reduction of the confounding effect of the instructor-student relation. Furthermore, given the synchronous, human-intensive nature of interviews, we argue that the presence of two reviewers during the interviews, with different roles, allowed us to capture a larger spectrum of behavior-related mistakes.

The current findings may be valid for group interviews performed in analogous settings, i.e., with a single customer, and with one or two projects. Furthermore, the majority of the students considered are non-native English speakers, conducting interviews in English. Different results may be obtained with native English speakers, and one-to-one interviews. Furthermore, the experiment was carried out by observations in only the first interview, therefore the results are entirely based on the commitment of students to have done the preparatory work before attending the interview.

\section{CONCLUSION AND FUTURE WORKS}

We have presented a research design for conducting an observational study of mistakes that novices make in requirements elicitation interviews. We also provided the results from the qualitative analysis of empirical data collected from multiple sources in this study. The significant number of mistakes observed and their classifications into 7 distinct themes provide a useful resource for educationists and trainers who wish to include elicitation interview training in their curriculum. Education research has shown that in general the two pedagogical approaches that we have utilized are effective for education and training, namely: role playing and authentic assessment. Our study reaffirms this in the context of REET. We believe that both of our contributions are not only useful in a university setting but also equally valuable in RE industry training.

Based on the results of our research, we are currently extending the study to all three interviews to observe how effective is corrective feedback approach to help students learn and improve their outputs. This could also generate additional guidance to students about where to exert more effort during RE process. We are also designing another study to extend our empirical research to the second assessment of this course which is a requirements inspection exercise in order to investigate the correlation between mistakes made in the interviews and the quality of the final SRS produced by students. In future, we plan to review not only the audio recordings but also the video recordings of the interviews for mistakes in non-verbal communications. Indeed, some mistakes associated with inappropriate and unprofessional behavior, e.g., arriving late, or not looking at the customer, can be clearly observed only with a video analysis. We argue that these mistakes may have a major impact on the rapport and trust-based relationship that the analyst is supposed to establish with the customer. 


\section{REFERENCES}

[1] Briggs, C.L., Learning how to ask: A sociolinguistic appraisal of the role of the interview in social science research. Vol. 1. 1986: Cambridge University Press.

[2] Zowghi, D. and C. Coulin, Requirements elicitation: A survey of techniques, approaches, and tools, in Engineering and managing software requirements. 2005, Springer. p. 19-46.

[3] Sutcliffe, A. and P. Sawyer. Requirements elicitation: Towards the unknown unknowns. in Requirements Engineering Conference (RE), 2013 21st IEEE International. 2013. IEEE.

[4] Davis, A., et al. Effectiveness of requirements elicitation techniques: Empirical results derived from a systematic review. in Requirements Engineering, 14th IEEE International Conference. 2006. IEEE.

[5] Dieste, O. and N. Juristo, Systematic review and aggregation of empirical studies on elicitation techniques. IEEE Transactions on Software Engineering, 2011. 37(2): p. 283-304.

[6] Donati, B., et al. Common Mistakes of Student Analysts in Requirements Elicitation Interviews. in International Working Conference on Requirements Engineering: Foundation for Software Quality. 2017. Springer.

[7] Pitts, M.G. and G.J. Browne, Improving requirements elicitation: an empirical investigation of procedural prompts. Information systems journal, 2007. 17(1): p. 89-110.

[8] Hogarth, R.M., et al., Learning from feedback: exactingness and incentives. Journal of Experimental Psychology: Learning, Memory, and Cognition, 1991. 17(4): p. 734.

[9] Li, S., The effectiveness of corrective feedback in SLA: A meta - analysis. Language Learning, 2010. 60(2): p. 309-365.

[10] Svensson, R.B. and B. Regnell, Is role playing in Requirements Engineering Education increasing learning outcome? Requirements Engineering, 2017. 22(4): p. 475-489.

[11] Zowghi, D. and S. Paryani. Teaching requirements engineering through role playing: Lessons learnt. in Requirements Engineering Conference, 2003. Proceedings. 11th IEEE International. 2003. IEEE.

[12] Yusop, N., Z. Mehboob, and D. Zowghi. The Role of Conducting Stakeholder Meeting in Requirements Engineering Techniques. in International Workshop on the Requirements Engineering Education and Training. 2007. IEEE Computer Society.

[13] Spoletini, P. and A. Ferrari. Requirements Elicitation: A Look at the Future Through the Lenses of the Past. in Requirements Engineering Conference (RE), 2017 IEEE 25th International. 2017. IEEE.

[14] Aranda, A.M., O. Dieste, and N. Juristo, Effect of domain knowledge on elicitation effectiveness: an internally replicated controlled experiment. IEEE
Transactions on Software Engineering, 2016. 42(5): p. 427-451.

[15] Hadar, I., P. Soffer, and K. Kenzi, The role of domain knowledge in requirements elicitation via interviews: an exploratory study. Requirements Engineering, 2014. 19(2): p. 143-159.

[16] Niknafs, A. and D. Berry, The impact of domain knowledge on the effectiveness of requirements engineering activities. Empirical Software Engineering, 2017. 22(1): p. 80-133.

[17] Niknafs, A. and D.M. Berry. An industrial case study of the impact of domain ignorance on the effectiveness of requirements idea generation during requirements elicitation. in Requirements Engineering Conference (RE), 2013 21st IEEE International. 2013. IEEE.

[18] Pitts, M.G. and G.J. Browne, Stopping behavior of systems analysts during information requirements elicitation. Journal of management information systems, 2004. 21(1): p. 203-226.

[19] Distanont, A., et al., The engagement between knowledge transfer and requirements engineering. International Journal of Management, Knowledge and Learning, 2012. 1(2): p. 131-156.

[20] Ferrari, A., P. Spoletini, and S. Gnesi, Ambiguity and tacit knowledge in requirements elicitation interviews. Requirements Engineering, 2016. 21(3): p. 333-355.

[21] Coughlan, J. and R.D. Macredie, Effective communication in requirements elicitation: a comparison of methodologies. Requirements Engineering, 2002. 7(2): p. 47-60.

[22] Agarwal, R. and M.R. Tanniru, Knowledge acquisition using structured interviewing: an empirical investigation. Journal of Management Information Systems, 1990. 7(1): p. 123-140.

[23] Browne, G.J. and M.B. Rogich, An empirical investigation of user requirements elicitation: Comparing the effectiveness of prompting techniques. Journal of Management Information Systems, 2001. 17(4): p. 223-249.

[24] Shuraida, S. and H. Barki, The influence of analyst communication in IS projects. Journal of the Association for Information Systems, 2013. 14(9): p. 482.

[25] Portugal, S., Interviewing users: how to uncover compelling details. Louis Rosenfeld, 2013.

[26] Lynda.com, Requirements Elicitation for Business Analysts: Interviews [Online]. https://www.lynda.com/Communicationtutorials/Requirements-ElicitationInterviews/410330-2.html.

[27] International, L.T., Developing User Requirements: The Key to Project Success," [Online]. https://www.learningtree.com/courses/315/developin g-user-requirements-training-the-key-to-projectsuccess/.

[28] Walcott-Justice, K., Requirements Elicitation: Artifact and Stakeholder Analysis," Coursera.com, [Online]. 
https://www.coursera.org/learn/requirementselicitation/lecture/FAN2U/stakeholder-elicitationstarting-interviewing-techniques.

[29] Hathaway, T.H.a.A., Requirements Elicitation Interviews and Workshops - Simply Put!: Best Practices, Skills, and Attitudes for Requirements Gathering on IT Projects. BA-Experts, 2016.

[30] Adams, S., Interviewing for journalists. 2001: Psychology Press.

[31] Martin, J.R., Actuality Interviewing and Listening: How to conduct successful interviews for nonfiction storytelling, actuality documentaries and other disciplines. 2017: Real Deal Press.

[32] Grobel, L., The Art of the Interview: Lessons from a Master of the Craft. 2010: Three Rivers Press.

[33] De Burgh, H., Skills are not enough: The case for journalism as an academic discipline. Journalism, 2003. 4(1): p. 95-112.

[34] DiCicco-Bloom, B. and B.F. Crabtree, The qualitative research interview. Medical education, 2006. 40(4): p. 314-321.

[35] Brinkmann, S., Interview, in Encyclopedia of critical psychology. 2014, Springer. p. 1008-1010.

[36] Jacob, S.A. and S.P. Furgerson, Writing interview protocols and conducting interviews: Tips for students new to the field of qualitative research. The Qualitative Report, 2012. 17(42): p. 1-10.

[37] Turner, D.I., Qualitative interview design: A practical guide for novice investigators. The qualitative report, 2010. 15(3): p. 754.

[38] Dilley, P., Conducting successful interviews: Tips for intrepid research. Theory into practice, 2000.39(3): p. 131-137.

[39] Seidman, I., Interviewing as qualitative research: $A$ guide for researchers in education and the social sciences. 2013: Teachers college press.

[40] Ritchie, J., et al., Qualitative research practice: $A$ guide for social science students and researchers. 2013: Sage.

[41] Morrison, J., The first interview. 2014: Guilford Publications.

[42] Warner, R.E., Solution-focused interviewing: Applying positive psychology, a Manual for Practitioners. 2013: University of Toronto Press.

[43] Miller, C., Interviewing strategies, in Diagnostic interviewing. 2003, Springer. p. 47-66.

[44] Hoffman, C.D., Investigative interviewing: strategies and techniques. International Foundation for Protection Officers, 2005.

[45] Investigations, I.f.I.C., "Investigative Interview Skills Course [Online]. https://iici.global/course/investigative-interviewskills-course, 2017.
[46] Ferrari, A., Spoletini, P., Donati, B., Zowghi, D., \& Gnesi, S. Interview Review: Detecting Latent Ambiguities to Improve the Requirements Elicitation Process. in Requirements Engineering Conference (RE), 2017 IEEE 25th International. 2017. IEEE.

[47] Spoletini, P., Ferrari, A., Bano, M., Zowghi, D., \& Gnesi, S Interview Review: An Empirical Study on Detecting Ambiguities in Requirements Elicitation Interviews. in International Working Conference on Requirements Engineering: Foundation for Software Quality. 2018. Springer.

[48] Burnay, C., I.J. Jureta, and S. Faulkner, What stakeholders will or will not say: A theoretical and empirical study of topic importance in Requirements Engineering elicitation interviews. Information Systems, 2014. 46: p. 61-81.

[49] Moody, J.W., J.E. Blanton, and P.H. Cheney, $A$ theoretically grounded approach to assist memory recall during information requirements determination. Journal of Management Information Systems, 1998. 15(1): p. 79-98.

[50] Wetherbe, J.C., Executive information requirements: getting it right. Mis Quarterly, 1991: p. 51-65.

[51] Pacheco, C. and I. Garcia, A systematic literature review of stakeholder identification methods in requirements elicitation. Journal of Systems and Software, 2012. 85(9): p. 2171-2181.

[52] Gervasi, V., et al., Unpacking tacit knowledge for requirements engineering, in Managing requirements knowledge. 2013, Springer. p. 23-47.

[53] Saiedian, H. and R. Dale, Requirements engineering: making the connection between the software developer and customer. Information and Software Technology, 2000. 42(6): p. 419-428.

[54] Lauer, T.W., E. Peacock, and S.M. Jacobs, Question generation and the systems analysis process. Questions and Information Systems, 1992: p. 47-61.

[55] Coughlan, J., M. Lycett, and R.D. Macredie, Communication issues in requirements elicitation: a content analysis of stakeholder experiences. Information and Software Technology, 2003. 45(8): p. 525-537.

[56] Gallivan, M.J. and M. Keil, The user-developer communication process: a critical case study. Information Systems Journal, 2003. 13(1): p. 37-68.

[57] Denzin, N.K. and Y.S. Lincoln, Handbook of qualitative research. 1994: Sage publications, inc. 